\title{
Stratification Factors
}

National Cancer Institute

\section{Source}

National Cancer Institute. Stratification Factors. NCI Thesaurus. Code C16153.

Selected pre-treatment factors by which patients are segreg ated to assure equal balance of these factors before randomization to the intervention arms of a clinical protocol. 